\title{
Streamlining screening of emotional function in Veterans with traumatic brain injury
}

Rull

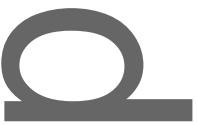

Jennifer M. Flaherty, Ph.D. ${ }^{1,2}$

Robert J. Spencer, Ph.D. ${ }^{2}$

Lauren L. Drag, Ph.D. ${ }^{3}$

Percival H. Pangilinan, M.D. ${ }^{2,4}$

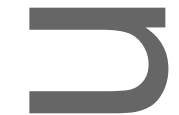

Linas A. Bieliauskas, Ph.D. ${ }^{2,4}$

1. Eastern Virginia Medical School, 2.Veterans Affairs Ann Arbor Healthcare System, 3.

Department of Neurology and Neurological Sciences, Stanford University Medical Center, 4.

University of Michigan Health System

Work performed at Veterans Affairs Ann Arbor Healthcare System

Corresponding Atthor: Jennifer M. Flaherty, EVMS Neuropsychology Program, 721 Fairfax Ave., Ste. 461, Norfolk, VA 23507; flaherjm@evms.edu; Phone: 757-446-8400; Fax: 757446-8401

Key Words. anxiety; depression; neurobehavioral; psychometric; sleep disturbance; traumatic brain injury

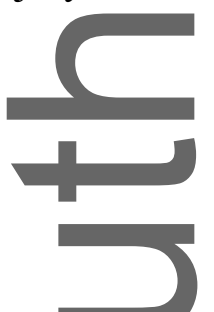

Objectives: This study examined how depression, anxiety, and sleep items from the Neurobehavioral Symptom Inventory (NSI) predict results from longer inventories. Method:

This is the author manuscript accepted for publication and has undergone full peer review but has not been through the copyediting, typesetting, pagination and proofreading process, which may lead to differences between this version and the Version of Record. Please cite this article as doi: $\underline{10.1002 / j \mathrm{clp} .22595 .}$.

This article is protected by copyright. All rights reserved. 
This was a retrospective review from 484, predominantly male (96.1\%) Veterans, mean age 29.7 years, who underwent brief neuropsychological screening during a comprehensive, multidisciplinary evaluation for mild traumatic brain injury (TBI). Participants completed the NSI, Insomnia Severity Index (ISI), and Hospital Anxiety and Depression Scale (HADS).

Results: $97.1 \%$ who endorsed "severe"/"very severe" anxiety on the NSI had significant anxiety on the HADS; 85\% reporting "severe"/"very severe" depression on the NSI, had significant depression on the HADS; and 97.7\% reporting "severe"/"very severe" sleep problems on the NSI, had significant sleep difficulties on the ISI. Conclusion: Close correspondence between "severe"/"very severe" symptoms on the NSI and lengthier checklists suggests additional checklists may be eliminated and individuals can be referred for mental-health treatment. NSI reports of "mild"/"moderate" require further screening.

\section{Introduction}

Referral for second-level comprehensive evaluation in Veterans Affairs (VA) polytrauma/traumatic brain injury (TBI) clinics is generally triggered when Veterans endorse symptomatic complaints following a head injury. In an effort to capture all individuals who may have possibly sustained a brain injury, TBI screenings were designed to cast a wide net (Lew et al.,2007). Hence, individuals with problems that may have occurred during the same event that caused their TBI, such as post-traumatic stress disorder (PTSD), cervico-cranial injury with headaches, inner ear injury (Spencer \& Adams, 2012), or nonspecific postconcussive symptoms often screen positive and also come to the attention of polytrauma/TBI clinics. Nevertheless, many individuals with TBI experience these conditions as well. As a result, many Veterans in polytrauma clinics may have TBI-like symptom complaints from a combination of TBI and non-TBI etiologies. Therefore, polytrauma evaluators are often faced with evaluating a wide range of psychiatric, psychosocial, and physical health concerns. 
Evaluators gather information on multiple aspects of psychosocial functioning in a circumscribed, often fixed, timeframe. Inherent in this process is the acknowledgement that psychological evaluation necessarily involves a tradeoff between speed and comprehensiveness. Traditional psychological assessments usually require significant time, but also provide in-depth information. When time is limited, however, targeted screening evaluations are more appropriate to the practical needs of assessment. Efficiency is particularly important in VA polytrauma/TBI clinics where multidisciplinary teams address many aspects of physical and mental health functioning within a single appointment. Therefore, efforts to streamline the evaluation, while still completing the necessary components (i.e., neurological, cognitive, mental health, case management), in a manner that is least burdensome to patients, are necessary to provide optimal patient centered care to Veterans. This study examined the use of psychiatric and neurobehavioral screening measures in a VA polytrauma/TBI clinic. Screening for mental health problems remains important because these individuals are statistically at higher risk for mental health comorbidity (Department of Veterans Affairs, 2007; Vanderploeg, Belanger, \& Curtiss, 2009), the most common of which are anxiety, PTSD, and depression (Belanger, Uomoto, \& Vanderploeg, 2009; Hoge et al., 2008; Kraal, Waldron-Perrine, Pangilinan, \& Bieliauskas, 2015; McCrae, 2008; Waldron-Perrine, Henrick, Spencer, Pangilinan, \& Bieliauskas, 2014), and there are empirically validated treatments for these diagnoses. Often, polytrauma/TBI clinics serve as a port of entry into the mental health system for Veterans.

Subjective self-impressions of depression, anxiety, and insomnia are commonly measured via objectively-scored checklists. The most popular of these measures range from 7-21 items (Bastien, Vallières, \& Morin; Beck \& Steer, 1987; Beck \& Steer, 1993; Radloff, 1977; Zigmond \& Snaith, 1983; Zung, 1965). For example, the HADS has been estimated to take 2-6 minutes to complete (Fitzpatrick, Gibbons, \& Mackintosh, 2009; Snaith, 2003) and 
the ISI has been estimated to take five minutes or less to complete (Smith \& Wegener, 2003). In the current sample, it has been observed, on average, that patients take approximately five minutes to complete the NSI. Therefore, such inventories are useful for efficiently quantifying symptom severity and monitoring symptomatic changes over time. Individually, these inventories are not generally onerous for most Veterans, but in combination they can be time-consuming and possibly repetitive. Additionally, individuals with brain injuries may experience more cognitive problems and fatigue more quickly, thus especially benefitting from more streamlined evaluations.

Instead of using separate checklists for each aspect of potential dysfunction in polytrauma populations, the VA requires the use of a single checklist that devotes mainly a single item to each problem. This checklist, the Neurobehavioral Symptom Inventory (NSI; Cicerone \& Kalmar, 1995), consists of 22 symptoms commonly reported by individuals following a concussion. The checklist includes physical, cognitive, and emotional aspects of functioning. Each item is rated on a five-point scale, with ratings of problems in each area ranging from "none," to "very severe." Factor analytic studies (Benger, Pastorek, \& Thornton, 2009; Caplan et al., 2010; Meterko et al., 2012; Vanderploeg et al., 2015) of the NSI in military and Veteran populations have been mixed, supporting three, four, and six factor solutions with the most consistent factors being cognitive; vestibular; affective; and somatie/sensory. Given that each area of functioning (e.g., depression) is covered by only one r. question, some clinicians supplement the NSI with lengthier self-report inventories, particularly for areas of interest such as depression, anxiety, PTSD, and sleep (Lew at al., 2007; Palmer et al., 2015).

A benefit of using single items to assess aspects of emotional functioning is brevity, but single items are often somewhat general and have less robust psychometric properties. 
Alternatively, other self-report screening measures contain more items of related content. As a result, multiple item self-report measures often have good psychometric properties such as fairly high internal consistency, as items tend to correlate highly with one another. Although longer self-report screening measures contain more items, the content is so conceptually similar that there will inevitably be a point of diminishing returns. Additional screening measures also take time that could otherwise be used to assess patients' needs. The tradeoff between the efficiency of employing single items and the added psychometric backing obtained by using screening instruments leads to an interesting practical question. Namely, among which categorizations of symptom severity are responses to single-items of emotional functioning sufficient as a screen for emotional concerns?

There is some support for the use of single items to assess depression in other settings. Williams et al.(1999) compared the use of a single question (i.e., "Have you felt depressed or sad much of the time in the past year?" to the Center for Epidemiological Studies Depression questionnaire (Radloff, 1977) in a community-based family medicine clinic, a VA general internal medicine clinic, and two university-affiliated general internal medicine clinics. They found that $41 \%$ answered yes to a single item. The sensitivity of the single item was $85 \%$ and specificity was $66 \%$.

Corson, Gerrity, and Dobscha (2004) conducted telephone screening of VA primary care patients using the above single item and the Patient Health Questionnaire (PHQ-9; Kroenke \& Spitzer, 2002). The majority of the 1211 participants $(80.3 \%)$ responded no to the single item and $19.7 \%$ responded yes. The majority of those who responded negatively to the single item also scored in the not depressed range on the PHQ-9 (77.1\%), but $14.7 \%$ scored in the mild range, $6 \%$ scored in the moderate range, $1.6 \%$ scored in the moderately severe range, and $0.6 \%$ scored in the severe range. For those with positive single item screens, 
$89.5 \%$ had at least mild depressive symptoms on the PHQ-9. The majority of patients

(30.7\%) with positive single item screens scored in the moderate range on the PHQ-9; $10.5 \%$ scored in the not depressed range, $17.6 \%$ scored in the mild range, $27.3 \%$ scored in the moderately severe range, and $13.9 \%$ scored in the severe range. Sensitivity for the single item using moderate severity on the PHQ-9 as the reference standard was $68 \%$ and specificity was 93\%. When moderately severe on the PHQ-9 was utilized as the reference standard, sensitivity increased and specificity decreased ( $82 \%$ and $87 \%$, respectively). The authors also compared the single item to the Diagnostic and Statistical Manual for Mental Disorders, $4^{\text {th }}$ Edition (DSM-IV; American Psychiatric Association, 1994) algorithm for major depressive disorder on the PHQ-9. This demonstrated $78 \%$ sensitivity and $88 \%$ specificity.

Blozik, Scherer, Lacruz, Ladwig, \& the KORA study group (2013) also compared the same yes/no single item question to the DSM-IV algorithm for depressive mood and major depressive disorder on the PHQ-9 in a community sample in Germany. $28 \%$ of participants were categorized as having depressive mood and $6.5 \%$ were classified as having major depressive disorder. Sensitivity of the single item compared to the depressive mood definition on the PHQ-9 was low (46.1\%) and specificity was high (94.3\%). Positive predictive value was $75.7 \%$ and negative predictive value was $81.8 \%$. Utilizing the major depressive disorder definition from the PHQ-9, the single item had $82.8 \%$ sensitivity, $87.5 \%$ specificity, $31.5 \%$ positive predictive value, and $98.7 \%$ negative predictive value.

This study explores the degree to which the depression, anxiety, and sleep items from the NSI predict screening results from other longer, commonly used, symptom inventories using sensitivity, specificity, and predictive power. Sensitivity is the proportion of true positives that a test correctly identifies. Specificity is the proportion of true negatives that a test identifies correctly (Altman \& Bland, 1994). This information can guide the clinician in 
selecting appropriate self-report measures to optimize efficiency and the quality of information gathered.

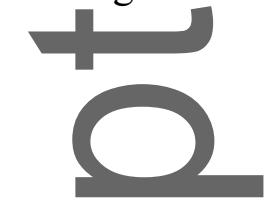

\section{Method}

\section{Participants}

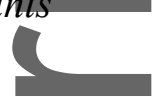

Archival data were obtained from a final sample of 484 Veterans, the majority of whom were referred for second-level evaluation (70.9\%), with a minority who were currently being evaluated for a TBI service connection claim (25.6\%), with 3.5\% for whom their status was not documented (i.e. whether or not they were being evaluated for a TBI service connection claim), at the polytrauma/TBI clinic of the Ann Arbor VA, a polytrauma support clinic team. Participants were consecutive or near consecutive referrals to the clinic. Participants completed the Hospital Anxiety and Depression Scale (HADS), Insomnia Severity Index, (ISI), and NSI as part of a brief neuropsychological screening battery. Individuals who scored $>22$ on the NSI Validity 10 (Vanderploeg et al., 2014), an embedded symptom validity measure, were eliminated from the analyses as over-reporting. This resulted in liminating $24.8 \%$ of the original sample of 644 participants. Participants in the final sample were $96.5 \%$ male, with an age range of 21 to 49 years (mean=29.7, standard deviation $(\mathrm{SD})=7.2$ ). The vast majority of the participants served in Operation Enduring Freedom (OEF), Operation Iraqi Freedom (OIF), or Operation New Dawn (OND), as is typical of VA polytrauma/TBI clinics, however, these identifying data were not entered into the database used and thus specific percentages are unavailable. While six individuals did not report their years of completed education, the mean level of education completed was 13 years $(\mathrm{SD}=2)$. The current sample included participants from other studies from this clinic (Boxley et al.,2016; Drag, Spencer, Walker, Pangilinan, Bieliauskas, 2012; Flaherty, Spencer, Drag, Pangilinan, \& Bieliauskas, 2015; Kraal et al., 2015; Spencer et al., 2013; Spencer, 
Drag, Walker, Pangilinan, \& Bieliauskas, 2010; Waldron-Perrine et al., 2014, WaldronPerrine et al., 2012).

Measures

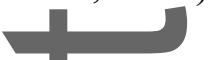

Measures

Neurobehavioral Symptom Inventory (NSI; Cicerone \& Kalmar, 1995). The NSI is a selfreport measure consisting of 22 items characteristic of postconcussive symptoms. The patient rates the severity of each symptom using a five-point scale as follows: $0=$ "none", $1=$ "mild", 2 "moderate", 3 = "severe", 4 = "very severe" based on functioning. Internal consistency of the total inventory has been demonstrated to be high in an OEF/OIF Veteran TBI sample ( $r=.95$; King et al., 2012). The current study examined specific items (r, s, and t) from the NSI that assess "difficulty falling or staying asleep"; "feeling anxious or tense"; and "feeling depressed or sad", respectively.

Hospital Anxiety and Depression Scale (HADS; Zigmond \& Snaith, 1983). The HADS consists of 14 items, seven of which are targeted to each measure depression or anxiety. Items are rated on a Likert-type scale from zero to three with the severity of most items rated according to how often symptoms occur. Total scores are calculated individually for the depression and anxiety items. Scores of 8-10 are indicative of mild symptoms, scores of 1114 indicate moderate symptoms, and scores of 15 or higher are classified as severe symptoms. Internal consistency of the total scale, as well as the anxiety subscale (HADS-A) and the depression subscale (HADS-D) is well-established, with reliability estimates exceeding 0.70 among non-diagnosed individuals (Crawford, Henry, Crombie, \& Taylor, 2001; Leung, Ho, Kan, Hung, \& Chen, 1993), medical patients (Golden, Conroy, \& O’Dwyer, 2007; Gough \& Hudson, 2009; Martin \& Thompson, 1999; Martin \& Thompson, 2000; Martin, Tweed, \& Metcalfe, 2004; McCue, Buchanan, \& Martin, 2006; McCue, Martin, Buchanan, Rodgers, \& Scholey, 2003; Stafford, Berk, \& Jackson, 2007; Sukantarat, 
Williamson, \& Brett, 2007; Woolrich, Kennedy, \& Tasiemski, 2006), and psychiatric patients (Cameron, Crawford, Lawton, \& Reid, 2008; Flint \& Rifat, 2002). Insomnia Severity Index (ISI; Bastien et al., 2001). The ISI is a seven item self-report inventory designed to measure the nature, severity, and impact of both daytime and nighttime components of insomnia within the past month. The dimensions evaluated are difficulty with sleep onset, sleep maintenance, and early morning awakening; sleep satisfaction; interference with daily functioning; noticeability of sleep problems by others; and distress. Each item is rated on a five point Likert-type scale from zero to four. The total score is interpreted as follows: $0-7$ absence of insomnia, 8-14 mild insomnia, 15-21 moderate insomnia, and 22-28 severe insomnia. Internal consistency was excellent in community and clinical samples (Cronbach $\alpha$ $=.90-.91$ ) and discriminant and convergent validity estimates were adequate to good (Morin, Belleville, Belanger, \& Ivers, 2011).

\section{Procedure}

This is a retrospective review of deidentified data from Veterans who also underwent brief neuropsychological screening as part of a comprehensive, multidisciplinary evaluation for mild TBI. All participants completed screening measures including the NSI, ISI, and HADS, among others (e.g., PTSD screening) prior to meeting with a clinician in order for clinicians to review the results before discussing them with participants. Veterans then met with clinical staff for a thorough clinical history, neurologic examination, psychosocial needs assessment/psychoeducation, and in most cases, neuropsychological screening. This investigation was approved by the Institutional Review Board at the Ann Arbor VA Healthcare System. 
Analyses were computed using SPSS Version 24 (IBM Corp., 2016). The data were screened for univariate and multivariate normality, linearity, and homoscedasticity. These analyses showed that univariate assumptions were met. Correlation coefficients between each NSI item and the total scores on the ISI, HADS-A, and HADS-D scales were computed. Item severity of each of the NSI items related to sleep, depression, and anxiety was compared to severity ranges on the ISI and HADS respectively. For the purposes of data analyses, NSI categories of "severe" and "very severe" were subsumed under the same category for crosstabulations to allow for a direct comparison, given that the longer screening measures included only one categorical descriptor in the severe range. When calculating sensitivity, specificity, and predictive power, NSI categories were dichotomized as "none/mild" versus "moderate" to "very severe" as has been done in previous research (Lew et al., 2011).

Correspondingly, HADS and ISI categories were also dichotomized as "none/mild" and "moderate/severe".

\section{Results}

The mean total NSI score was $36.5(\mathrm{SD}=13.7)$. The mean scores for the depression, anxiety, and sleep items were 1.8 $(\mathrm{SD}=1.3), 2.4(\mathrm{SD}=1.2)$, and $2.7(\mathrm{SD}=1.2)$, respectively. The mean score for the depression item was in the mild range and the mean scores for the anxiety and sleep items were in the moderate range. Mean scores for the ISI (mean= 15.8 and $\mathrm{SD}=6.4)$, HADS-A (mean=11.4, $\mathrm{SD}=4.1)$ and HADS-D $($ mean= 8.5, $\mathrm{SD}=4.3)$ were each above the clinically meaningful cutoff score (i.e., $>7$ for each measure). More specifically, the mean ISFand HADS-A scores were in the moderate range, and the mean HADS-D score was in the mild range. Correlations between the NSI items and total scores on the HADS-A, HADS-D, and ISI are reported in Table 1. Briefly, the vast majority of these correlations 
were significant, the exception was the item about hearing difficulty. The strongest correlations were between each item and its corresponding longer measure.

Overall, participants' reports of symptoms on the NSI agreed with the categorical descriptions provided by the HADS and ISI on 35-44\% of occasions. Looking at the individualitems, scores for the NSI anxiety item fell within the same descriptive category on the HADS-A in $44.2 \%$ of cases. Scores for the NSI depression item fell within the same descriptive category on the HADS-D in $34.9 \%$ of cases. Scores on the NSI sleep item fell within the same-descriptive category for the ISI in $36.2 \%$. Tables 2 through 4 display individuals' responses on the NSI items, and how these correspond to the severity of scores on the parallel screening measure.

We defined positive NSI and HADS-A as indicating at least moderate endorsement. Results indicated that the NSI item had specificity of $48.4 \%$ and sensitivity of $94.6 \%$. This (n) cutoff produced a positive predictive value of $74.3 \%$, negative predictive value of $85.1 \%$, and an overall predictive value of $76.7 \%$.

Among the 241 participants who indicated "severe" or "very severe" anxiety on the NSI, $97.1 \%$ scored in the clinically significant range on the HADS-A (11.6\% mild, $42.7 \%$ each for moderate and severe). The results of comparisons between "mild" and "moderate" severity ratings on the NSI and HADS-A score were less clear, showing less correspondence between measures. Among the 69 participants who reported "mild" anxiety on the NSI, the majority of participants $(46.4 \%)$ were below the clinical cutoff on the HADS-A, followed by $34.8 \%$ who seored in the mild range, $17.4 \%$ in the moderate range, and $1.4 \%$ in the severe range. Among the 136 participants who reported "moderate" anxiety on the NSI, the majority of participants (47.8\%) also scored in the moderate range on the HADS-A, but $14.7 \%$ were 
below the clinical cutoff, $30.9 \%$ scored in the mild range, and $6.6 \%$ scored in the severe range.

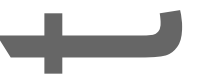

The specificity of the NSI depression item when compared to the HADS-D was $55.6 \%$ and the sensitivity was $83.9 \%$. The positive predictive value was $47.1 \%$, negative predictive value was $88 \%$, and the overall predictive value was $64.7 \%$.

Among the 160 participants who reported "severe" or "very severe" depression on the NSI, $85 \%$ scored in the clinically significant range on the HADS-D $(24.4 \%$ mild, $43.8 \%$ moderate and $16.9 \%$ severe). The results of comparisons between "mild" and "moderate" severity ratings on the NSI and HADS-D score were again less clear and showed less correspondence between measures. Among the 99 participants who reported "mild" depression on the NSI, the majority (50.5\%) were below the clinical cutoff on the HADS-D, followed by $30.3 \%$ who scored in the mild range, $16.2 \%$ in the moderate range, and $3 \%$ in the severe range. Among the 116 participants who reported "moderate" depression on the NSI, the majority of participants (41.4\%) scored in the mild range on the HADS-D, followed by $30.2 \%$ below the clinical cutoff, $23.3 \%$ in the moderate range, and $5.2 \%$ in the severe range.

Comparisons of the NSI sleep item with the ISI yielded a specificity of $39.4 \%$ and sensitivity of $97 \%$. The positive predictive value was $71.6 \%$, negative predictive value was $89.2 \%$, and overall predictive value was $74.6 \%$.

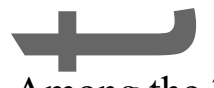

Among the 306 participants who reported "severe" or "very severe" sleep problems on the NSI, $97.7 \%$ scored in the clinically significant range on the ISI ( $14.4 \%$ mild, $52.9 \%$ moderate and $30.4 \%$ severe). Among those reporting no sleep problems on the NSI item, $24.2 \%$ of participants indicated mild or moderate sleep problems on the ISI $(21.2 \%$ mild, $3 \%$ moderate). The results of comparisons between "mild" and "moderate" severity ratings on the NSI and ISI were less clear, showing less correspondence between measures. Among the 50 
participants who reported "mild" sleep difficulties on the NSI, the majority of participants $(50 \%)$ also scored in the mild range on the ISI, however $34 \%$ were below the clinical cutoff, $14 \%$ scored in the moderate range, and $2 \%$ scored in the severe range. Among the 95 participants who reported "moderate" sleep difficulties on the NSI, the majority of participants $(51.6 \%)$ scored in the mild range on ISI, followed by 33.7 in the moderate range, $14.7 \%$ below the clinical cutoff, and $0 \%$ in the severe range.

\section{$\circlearrowleft$}

\section{Discussion}

Anxiety, depression, and sleep problems are frequently reported by individuals undergoing polytrauma evaluation for suspected TBI. In this sample, the self-reported categorical descriptors from the NSI agreed with the categorical descriptions provided by lengthier self-report inventories on $35-44 \%$ of occasions. The rate of agreement was particularly low for "mild" and "moderate" ratings on the NSI. This rate of agreement may be ( viewed as somewhat low, and may be, in part, related to the different interpretive ranges used among the measures. Therefore, the discussion focuses on "none", "severe", and "very severe" ratings on the NSI for which there was close correspondence with the lengthier selfreport checklists. Veterans reporting "severe" or "very severe" psychiatric and sleep problems on the NSI nearly always indicated clinically significant psychiatric problems on lengthier inventories. This suggests that it is reasonable for clinicians in TBI clinics to forgo additional screening checklists in these individuals because checklists do not provide unique information in the overwhelming number of cases. If over-reporting has been ruled out using embedded or stand-alone measures of symptom validity, these individuals should be referred for mentat health treatment. Additionally, when individuals indicated "none" on the NSI, only $3-7.9 \%$ of individuals reported more than mild symptoms on the longer inventories. Therefore, additional screening measures need not be administered in these cases either, but 
follow up questions regarding mental health symptoms and treatment can be addressed during the psychiatric and psychosocial clinical interview and triaged as needed.

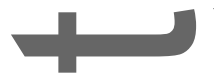

In contrast, there was a good degree of variability in self-reported psychiatric symptoms on longer inventories among Veterans indicating mild or moderate psychiatric problems on the NSI. In these cases, the longer screening inventories do assist in further characterizing their mental health problems and potentially add incremental information to the assessment and may avoid sending individuals for further mental health evaluation who do not need it and thereby conserve mental health resources.

Regarding sensitivity, specificity, and predictive power for the NSI depression item compared to the HADS-D, results of the current study were relatively similar to previous research, particularly sensitivity. Blozik et al. (2013), Corson et al. (2004), and Williams et al. (1999) reported sensitivity ranging from $82-85 \%$ when more stringent criteria were used, compared to the present finding of $83.9 \%$. Specificity in the current study was lower than in prior research (55.6\% vs. 66-94.3\%). However, it can be argued that in depression screening, sensitivity is more critical in order not to miss anyone with possible depression, which is a risk factor for suicide. Findings for predictive power were more variable between studies. Blozik et al.(2013) found $75.7 \%$ positive predictive power and $81.8 \%$ negative predictive power when the less stringent depressive mood classification was utilized, versus $31.5 \%$ positive predictive power and $98.7 \%$ negative predictive power when the major depressive disorder classification was used. Results from the current student fell between these values with $47.1 \%$ positive predictive power and $88 \%$ negative predictive power.

For the $33.1 \%$ to $63.2 \%$ of individuals reporting "severe" or "very severe" anxiety, depression, or sleep problems on the NSI, additional screening with face-valid self-report scales can be dispensed with, as screening has already served its purpose. These results can 
lead to procedures that enhance the efficiency of psychological assessment through the use of an algorithm for initial screening. This approach would help divert from routine and perfunctory completing of checklists, towards investment in individuals most likely in need of further assessment and treatment. As a result, the burden to patients is reduced; this may be especially important for individuals with TBI who may have difficulties reporting symptoms and with the length of the evaluation. If participants in the current study who endorsed, none, severe, or very severe anxiety, depression, and sleep problems on the NSI were not required to complete the HADS and ISI, this would have resulted in decreased burden and effort (e.g., approximately 11 minutes) for them and save five minutes of clinicians' time.

Given the high frequency of psychiatric complaints among those seeking care for TBI (Department of Veterans Affairs, 2007; Kraal et al., 2015; Waldron-Perrine, et al., 2014), polytrauma clinios are in the position of serving as ports of mental health linkage for our Veterans. Regardless of the route of referral, or the length of time since injury, Veterans undergoing TBI assessment tend to report a high degree of psychiatric symptomatology (Department of Veterans Affairs, 2007; Kraal et al., 2015; Waldron-Perrine, et al., 2014). NSI results can assist clinicians in polytrauma clinics in triaging Veterans for mental health care.

A limitation of this study is that the results are specific to VA polytrauma/TBI clinics, and more specifically, to the demographic composition of this sample, that is, predominantly Caucasian males in their 20s-30s who served in OEF, OIF, or OND. Future research should expand the sample to include more females and other settings such as mental health clinics and settings outside of the VA. Additionally, replicating this study in other, non-TBI, patient samples could shed more light on why, in some cases, there were low percentages of agreement between the NSI and the HADS and ISI. 
Another limitation is that the NSI does not have any items that directly assess PTSD symptoms. This is an important limitation as PTSD is one of the most frequently comorbid mental health condition among VA polytrauma patients (Belanger et al., 2009; Hoge et al., 2008), thus an additional screening measure for PTSD would be necessary in conjunction with the NSI. Future research could examine how items on the NSI (e.g., "feeling anxious or tense", "difficulty falling or staying asleep", "forgetfulness, can't remember things", "poor concentration, can't pay attention, easily distracted", "feeling depressed or sad", and “irritability, easily annoyed") compare to a questionnaire or structured interview for PTSD symptoms.

Although this study eliminated those individuals demonstrating over-reporting as indicated by a cutoff of $>22$ on the NSI Validity 10 as recommended by Vanderploeg et al. (2014), other researchers (Lange, Brickell, \& French, 2015; Lange, Brickell, Lippa, \& French, 2015) have utilized lower cutoffs. The NSI Validity 10 is a relatively new scale, and needs further replication. Therefore, there may still have been some individuals who overreported in the current sample.

Finally, this study used self-report measures, which have inherent weaknesses such as difficulty or bias in recalling or reporting of symptoms that must be compensated for with other assessment methods including clinical interview, behavioral observation, informant report, medical record review, and/or psychological testing. Self-report measures are often moderately correlated with one another, as they were in this study, and this may be partially due to the non-independence of these measures. While, this could have led to higher false positive rates, non-independence is common in clinical situations and cannot be avoided; therefore, these results likely accurately reflect clinicians' experiences. 
Further research is needed to determine if severe and very severe ratings on the NSI also fulfill the screening function for other commonly used self-report face-valid measures and reference standards such as a structured interview. Additional research should further explore the situations for which narrow-band screening measures, such as the HADS or ISI, provide incremental information beyond very terse instruments like the NSI.

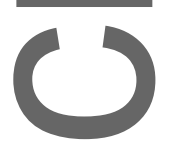

\section{Conclusion}

In conclusion, when conducting brief psychological screening in the context of interdisciplinary medical and psychosocial evaluations, a report of "severe" or "very severe" distress on single-item questions of depression, anxiety, and sleep problems on the NSI can provide adequate information for triage. Additional initial screening is likely unnecessary for these Veterans and is unlikely to yield incrementally valuable information. Instead, these individuals can be referred for mental health treatment for depressive and anxiety symptoms or sleep diffieulties on the basis of their severe NSI scores. For individuals reporting less than "severe" problems, additional screening in TBI clinics provides useful additional information regarding psychological distress.

\section{Acknowledgements}

The authors report no conflicts of interest. The present study was approved by the Institutional Review Board as part of a larger data collection and was completed in accordance with the Code of Ethics of the World Medical Association (Declaration of Helsinki).

$$
\text { i). }
$$

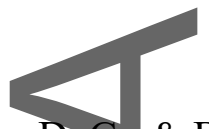

\section{References}

Altman, D. G., \& Bland, J. M. (1994). Statistics Notes: Diagnostic tests 1: sensitivity and specificity British Medical Journal, 308, 1552. DOI: 10.1136/bmj.308.6943.1552 
American Psychiatric Association (1994). Diagnostic and Statistical Manual of Mental Disorders (4 ${ }^{\text {th }}$ Ed.). Washington, DC: Author.

(1)

Bastien, C. H., Vallières, A., \& Morin, C.M. (2001). Validation of the Insomnia Severity Index as an outcome measure for insomnia research. Sleep Medicine, 2, 297-307.

Beck, A. F., \& Steer, R. A. (1987). Manual for the revised Beck Depression Inventory. San Antonio: Psychological Corporation.

Beck, A. T.Steer, R. A. (1993). Beck Anxiety Inventory Manual. San Antonio: Psychological Corporation Harcourt Brace \& Company.

Belanger, H. G., Uomoto J. M., Vanderploeg, R.D. (2009). The Veterans Health Administration System of Care for Mild Traumatic Brain Injury: Costs, Benefits, and Controversies. Journal of Head Trauma Rehabilitation, 24(1), 4-13.

Benge, J. F., Pastorek, N. J., \& Thornton, G. M. (2009). Postconcussive symptoms in OEFOIF veterans: factor structure and impact of posttraumatic stress. Rehabilitation Psychology, 54, 270-278.

Blozik, E., Scherer, M., Lacruz, M. E., Ladwig, K., \& the LORA study group (2013). Diagnostic utility of a one-item question to screen for depressive disorders: Results from the LORA F3 study. BioMed Central Family Practice, 14(198), 1-7. DOI: 10.1186/1471-2296-14-198

Boxley, L., Flaherty, J. M., Spencer, R. J., Drag, L. L., Pangilinan, P. H., \& Bieliauskas, L. A. (2016). Reliability and factor structure of the Hospital Anxiety and Depression Scale among Veterans suspected of traumatic brain injury. Journal of Rehabilitation Research \& Development, 53(6), 873-880. DOI: 10.1682/JRRD.2015.05.0088 
Cameron, I. M., Crawford, J. R., Lawton, K., \& Reid, I. C. (2008). Psychometric comparison of PHQ-9 and HADS for measuring depression severity in primary care. British Journal of General Practice, 58(546), 32-36.

Caplan, L., Ivins, B., Poole, J. H., Vanderploeg, R D., Jaffee, M. S., \& Schwab, K. (2010). The structure of postconcussive symptoms in three U.S. military samples. Journal of Head Trauma Rehabilitation, 25(6), 447-458.

Cicerone, K., \& Kalmar, K. (1995). Persistent postconcussion syndrome: The structure of subjective complaints after mild traumatic brain injury. Journal of Head Trauma Rehabilitation, 10(3), 1-17.

Corson, K., Gerrity, M. S., \& Doscha, S. K. (2004). Screening for depression and suicidality in a VA primary care setting: 2 items are better than 1 item. The American Journal of Managed Care, 10(11), 845.

Crawford, J.R., Henry, J. D., Crombie, C., \& Taylor, E. P. (2001). Normative data for the HADS from a large non-clinical sample. British Journal of Clinical Psychology, 40, $429-434$.

Department of Veterans Affairs. (2007). Screening and evaluation of possible traumatic brain injury in Operation Enduring Freedom (OEF) and Operation Iraqi Freedom (OIF) Veterans. Washington (DC): Department of Veterans Affairs Veterans Health Administration.

Drag, L. L., Spencer, R. J., Walker, S. J., Pangilinan, P. H., \& Bieliauskas, L. A. (2012). The Contributions of Self-Reported Injury Characteristics and Psychiatric Symptoms to Cognitive Functioning in OEF/OIF Veterans with Mild Traumatic Brain Injury. Journal of the International Neuropsychological Society, 18, 576-584. 
Fitzpatrick, R., Gibbons, E., \& Mackintosh, A. (2009). An overview of patient-reported outcome measures for people with anxiety and depression 2009. Retrieved from http://phi.uhce.ox.ac.uk/pdf/Depression\%20and\%20Anxiety\%20PROMGroup\%20Ox ford $\% 20$ may2010.pdf

Flaherty, J. M., Spencer, R. J., Drag, L. L., Pangilinan, P. H., \& Bieliauskas, L. A. (2015). Limited usefulness of the Rey Fifteen- Item Test in detection of invalid performance in Veterans suspected of mild traumatic brain injury. Brain Injury. DOI:

$10.3109 / 02699052.2015 .1075249$

Flint, A. J., \& Rifat, S. L. (2002). Factor structure of the hospital anxiety \& depression scale in older patients with major depression. International Journal of Geriatric Psychiatry, 17, 117-123.

Golden, J., Conroy, R. M., \& O'Dwyer, A. M. (2007). Reliability and validity of the hospital anxiety and depression scale and the beck depression inventory (full and fast screen scales). Journal of Affective Disorders, 100, 265-269.

Gough, K., \& Hudson. P. (2009). Psychometric properties of the hospital anxiety and depression scale in a family of caregivers of palliative care patients. Journal of Pain Symptom Management, $37,797-806$.

Hoge, C. W., McGurk, D. M., Thomas, J. L., Cox, A. L., Engel, C. C., \& Castro, C. A. (2008). Mild traumatic brain injury in the U.S. soldiers returning from Iraq. The New England Journal of Medicine, 358(5), 453-463.

IBM Corp. (2016). IBM SPSS Statistics for Windows, Version 24.0. Armonk, NY: IBM Corp.

King, P. R., Donnelly, K.T., Donnelly, J. P., Dunnam, M., Warner, G., Kittleson, C. J.,...Meier, S. T. (2012). Psychometric study of the Neurobehavioral Symptom Inventory. Journal of Rehabilitation Research and Development, 49(6), 879-888. 
Kraal, A. Z., Waldron-Perrine, B., Pangilinan, P. H., Bieliauskas, L. A. (2015). Affect and psychiatric symptoms in a Veteran polytrauma clinic. Rehabilitation Psychology, 60(1), 36-42.

Kroenke, K.\& Spitzer, R. L. (2002). The PHQ-9: A new depression diagnostic and severity measure. Psychiatric Annals, 32(9), 1-7.

Lange, R. T., Brickell, T. L., \& French, L. M. (2015). Examination of the Mild Brain Injury Atypical Symptom Scale and the Validity-10 Scale to detect symptom exaggeration in US military service members. Journal of Clinical and Experimental Neuropsychology, 37(3), 325-337. DOI: 10.1080/13803395.2015.1013021

Lange, R. T., Brickell, T. L., Lippa, S. M., \& French, L. M. (2015). Clinical utility of the Neurobehavioral Symptom Inventory validity scales to screen for symptom exaggeration following traumatic brain injury. Journal of Clinical and Experimental Neuropsychology, 37(8), 853-862. DOI: 10.1080/13803395.2015.1064864

Leung, C. M., Ho, S., Kan, C.S., Hung, C.H., \& Chen, C.N. (1993). Evaluation of the Chinese version of the hospital anxiety and depression scale: A cross cultural perspective. International Journal of Psychosomatics, 40, 29-34.

Lew, H. L.,Pogoda, T. K., Baker, E., Stolzmann, K. L., Meterko, M., Cifu D. X.,... Hendricks, A. M. (2011). Prevalence of dual sensory impairment and its association with traumatic brain injury and blast exposure in OEF/OIF veterans. Journal of Head Trauma Rehabilitation, 26(6), 489-496.

Lew, H. L., Poole, J. H., Vanderploeg, R. D., Goodrich, G. L., Dekelboum, S., Guillory, S. B.,...Cifu, D. X. (2007). Program development and defining characteristics of returning military in a VA polytrauma network site. Journal of Rehabilitation Research and Development, 44(7), 1027-1034. 
Martin, C. R., \& Thompson, D. R. (1999). Utility of the hospital anxiety and depression scale in patients with end-stage renal disease on continuous ambulatory peritoneal dialysis. Psychological Health Medicine, 4, 369-376.

Martin, C. R., \& Thompson, D. R. (2000). A psychometric evaluation of the hospital anxiety and depression scale in coronary care patients following acute myocardial infarction. Psychological Health Medicine, 5, 193-201.

Martin, C. R., Tweed, A. E., \& Metcalfe, M. S. (2004). A psychometric evaluation of the hospital anxiety and depression scale in the patients diagnosed with end-stage renal disease. British Journal of Clinical Psychology, 43, 51-64.

McCrae, M. (2008). Mild traumatic brain injury and postconcussion syndrome: The new evidence base for diagnosis and treatment. New York: Oxford.

McCue, P., Buchanan, T., \& Martin, C. R. (2006). Screening for psychological distress using internet administration of the hospital anxiety \& depression scale (HADS) in individuals with chronic fatigue syndrome. British Journal of Clinical Psychology, 45, 483-498.

McCue, P., Martin, C. R., Buchanan, T., Rodgers, J, \& Scholey, A. B. (2003). An investigation into the psychometric properties of the hospital anxiety and depression scale (HADS) in individuals with chronic fatigue syndrome. Psychological Health Medicine, 8(4), 425-439.

Meterko, M.., Bakerr, E., Stolzmann, K. L., Hendricks, A. M., Cicerone, K. D., \& Lew, H. L. (2012). Psychometric Assessment of the Neurobehavioral Symptom Inventory-22: The structure of persistent postconcussive symptoms following deployment-related mild traumatic brain injury among veterans. Journal of Head Trauma Rehabilitation, 27(1), 55-62. 
Morin, C. M., Belleville, G., Belanger, L., \& Ivers, H. (2011). The Insomnia Severity Index:

Psychometric indicators to detect insomnia cases and evaluate treatment response. SLEEP, 34(5), 601-608.

Palmer, G. A., Happe, M. C., Paxson, J. M., Jurek, B. K. W., Graca, J. J., \& Olson, S. A. (2015).

Psychological predictors of neurobehavioral symptoms in a polytrauma veteran sample.

Journal of Loss and Trauma. DOI:10.1080/15325024.2015.1072009

Radloff, L. \$. (1977). The CES-D scale: a self-report depression scale for research in the general population. Applied Psychological Measurement, 1, 385-401.

Smith, M. T., \& Wegener, S. T. (2003). Measures of sleep: The Insomnia Severity Index, Medical Outcomes Study (MOS) Sleep Scale, Pittsburgh Sleep Diary (PSD), and Pittsburgh Sleep Quality Index (PSQI). Arthritis \& Rheumatism, 49(5S), S184-S196.

Snaith, R. P. (2003). The Hospital Anxiety and Depression Scale. Health and Quality of Life Outcomes, 1(29). DOH: $10.1186 / 1477-7525-1-29$

Spencer, R.J., \& Adams, K. A. (2012). Neuropsychology and polytrauma services. In: S. S. Bush (Ed.), Neuropsychological practice with Veterans (pp. 77-98). New York: Springer Publishing

\section{Company.}

Spencer, R. J., Axelrod, B. N., Drag, L. L., Waldron-Perrine, B., Pangilinan, P. H., Bieliauskas, L. A. (2013). WAIS-IV Reliable Digit Span is no More Accurate than Scaled Score as an Indicator of Invalid Performance in a Veteran Sample Undergoing Evaluation for mTBI. The Clinical Neuropsychologist, 27(8), 1362-1372.

Spencer, R. J, Drag, L.L., Walker, S. J., Pangilinan, P. H., \& Bieliauskas, L. A. (2010). Selfreports of cognitive function are not predictive of neuropsychological test 
performance among returning combat Veterans. Journal of Rehabilitation Research and Development, 47, 521-530.

Stafford, L., Berk, M., \& Jackson, H. J. (2007). Validity of the hospital anxiety and depression scale and patient health questionaaire-9 to screen for depression in patients with coronary artery disease. General Hospital Psychiatry, 29, 417-424.

Sukantarat, K. T., Williamson, R. C. N., \& Brett, S. J. (2007). Psychological assessment of ICU survivors: A comparison between the Hospital Anxiety \& Depression Scale and the Depression, Anxiety, and Stress scale. Anaesthesia, 62, 239-243.

Vanderploeg, R. D., Belanger, H. G., \& Curtiss, G. (2009). Mild traumatic brain injury and posttraumatic stress disorder in their associations with health symptoms. Archives of Physical Medicine and Rehabilitation, 90(7), 1084-1093.

Vanderploeg, R. D., Cooper, D. B., Belanger, H. G., Donnell, A. J., Kennedy, J. E., Hopewell, C. A., \& Scott, S. G. (2014). Screening for postdeployment conditions: Development and cross-validation of an embedded validity scale in the Neurobehavioral Symptom Inventory. Journal of Head Trauma Rehabilitation, 29(1), 1-10.

Vanderploeg, R. D., Silva, M. A., Soble, J. R., Curtiss, G., Belanger, H. G., Donnell, A. J., \& Scott, S. G. (2015). The structure of postconcussion symptoms on the Neurobehavioral Symptoms Inventory: A comparison of alternative models. Journal of Head Trauma Rehabilitation, $30(1), 1-11$.

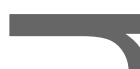

Waldron-Perrine, B., Henrick, H., Spencer, R., Pangilinan, P., \& Bieliauskas, L. (2014). Post concussive symptom report in polytrauma: Influences of mild traumatic brain injury and psychiatric disorders. Military Medicine, 179(8), 856-864. 
Waldron-Perrine, B., McGuire, A. P., Spencer, R. J., Drag, L. L., Pangilinan, P. H., \& Bieliauskas, L. A. (2012). The influence of sleep and mood on cognitive functioning

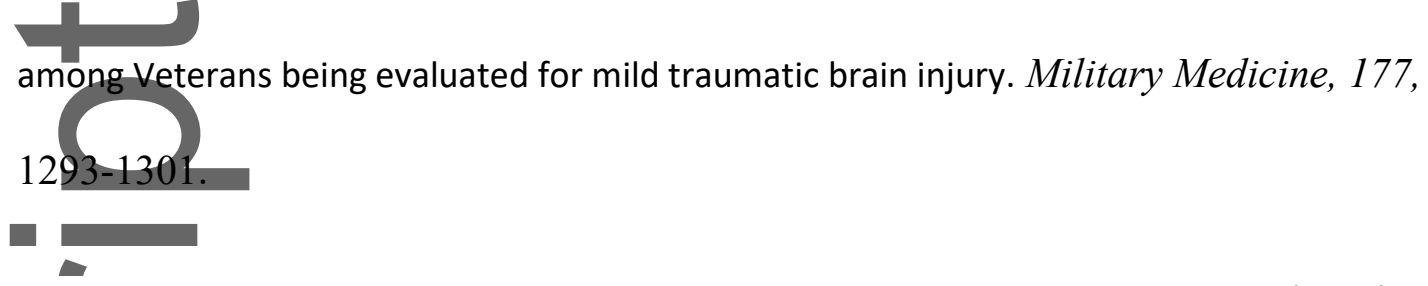

Williams, J. W., Mulrow, C. D., Kroenke, K., Dhanda, R., Badgett, R. G., Omori, D., \& Lee, S. (1999). Case-Finding for depression in primary care: A randomized trial. The American Journal of Medicine, 106, 36-43.

Woolrich, R. A., Kennedy, P., \& Tasiemski, T. (2006). A preliminary psychometric evaluation of Hospital Anxiety and Depression Scale (HADS) in 963 people living with a spinal cord injury. Psychological Health Medicine, 11(1), 80-90.

Zigmond, A. S., \& Snaith, R. P. (1983). The hospital anxiety and depression scale. Acta Psychiatrica Scandinavica, 67, 361-370.

Zung, W. W. (1965). A self-rating depression scale. Archives of General Psychiatry, 12, 63-70.

Table 1. Correlations between NSI items, HADS subscale totals, and ISI total score

\begin{tabular}{lccc}
\hline & HADS-D & HADS-A & ISI Total \\
\hline Dizzy & $.21^{* * *}$ & $.19^{* * *}$ & $.20^{* * *}$ \\
Balance & $.32^{* * *}$ & $.22 * * *$ & $.20^{* * *}$ \\
Coordination & $.26^{* * *}$ & $.24^{* * *}$ & $.24^{* * *}$ \\
Headaches & $.19^{* * *}$ & $.16^{* *}$ & $.25^{* * *}$ \\
Nausea & $.28^{* * *}$ & $.22^{* * *}$ & $.25^{* * *}$ \\
Vision &. $.26^{* * *}$ & $.20^{* * *}$ & $.27^{* * *}$ \\
Light Sensitivity &. $.21^{* * *}$ & $.16^{* *}$ & $.19^{* * *}$ \\
Hearing &. .08 & .07 & $.11^{*}$ \\
Noise Sensitivity & $.20^{* * *}$ & $.24 * * *$ & $.21^{* * *}$ \\
Numbness/Tingling &. $.22^{* * *}$ & $.25^{* * *}$ & $.35^{* * *}$
\end{tabular}

This article is protected by copyright. All rights reserved. 


\begin{tabular}{|c|c|c|c|}
\hline Taste/Smell & $.15^{* *}$ & $.13^{*}$ & $.15^{* *}$ \\
\hline Loss/Increase Appetite & $.35 * * *$ & $.28 * * *$ & $.29 * * *$ \\
\hline Concentration/Attention & $.37 * * *$ & $.37 * * *$ & $.33 * * *$ \\
\hline Forgetfuln & $.36^{* * *}$ & $.25 * * *$ & $.30 * * *$ \\
\hline ring Decisions & $.40 * * *$ & $.33 * * *$ & $.28 * * *$ \\
\hline Slowed Thii & $.42 * * *$ & $.34 * * *$ & $.31 * * *$ \\
\hline Fatigue & $.42 * * *$ & $.27 * * *$ & $.40 * * *$ \\
\hline Sleep & $.37 * * *$ & $.38 * * *$ & $.71 * * *$ \\
\hline Anxious & $.41 * * *$ & $.65 * * *$ & $.40 * * *$ \\
\hline Depressed & $.59 * * *$ & $.50 * * *$ & $.41 * * *$ \\
\hline Irritability & $.45 * * *$ & $.51 * * *$ & $.44 * * *$ \\
\hline Frustration/overwhelmed & $.44 * * *$ & $.54 * * *$ & $.40 * * *$ \\
\hline NSI Total Score & $.57 * * *$ & $.53 * * *$ & $.55 * * *$ \\
\hline
\end{tabular}

Note: ${ }^{*} \mathrm{r}<.05 ;{ }^{*} \mathrm{r}<.01 ; * * * \mathrm{r}<.001 ;$ HADS $=$ Hospital Anxiety and Depression Scale;

HADS-A = HADS anxiety subscale, HADS-D = HADS depression subscale; ISI = Insomnia Severity Index; NSI = Neurobehavioral Symptom Inventory; Bolded items and correlations correspond with the criterion scale

Table 2. Correspondence between NSI sleep item and ISI severity

\begin{tabular}{lcccccc}
\hline \multicolumn{5}{c}{ NSI “Difficulty falling or staying asleep” Severity } & \\
& & & & & \\
& & & & & & \\
\hline
\end{tabular}

Note: ISI = Insomnia Severity Index; NSI = Neurobehavioral Symptom Inventory

This article is protected by copyright. All rights reserved. 


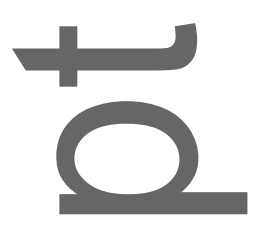

Table 3. Correspondence between NSI anxiety item and HADS-A severity

\begin{tabular}{lllllll}
\hline & & & \\
\hline
\end{tabular}

Note: HADS-A = Hospital Anxiety and Depression Scale, anxiety subscale; NSI = Neurobehavioral Symptom Inventory

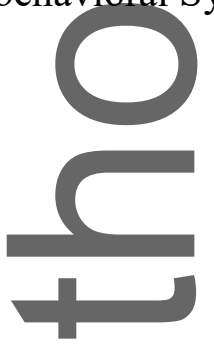

Table 4. Correspondence between NSI depression item and HADS-D severity

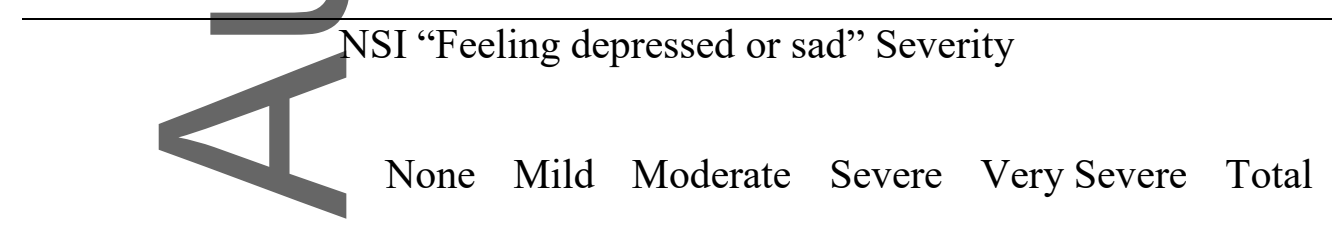

HADS-D Severity 


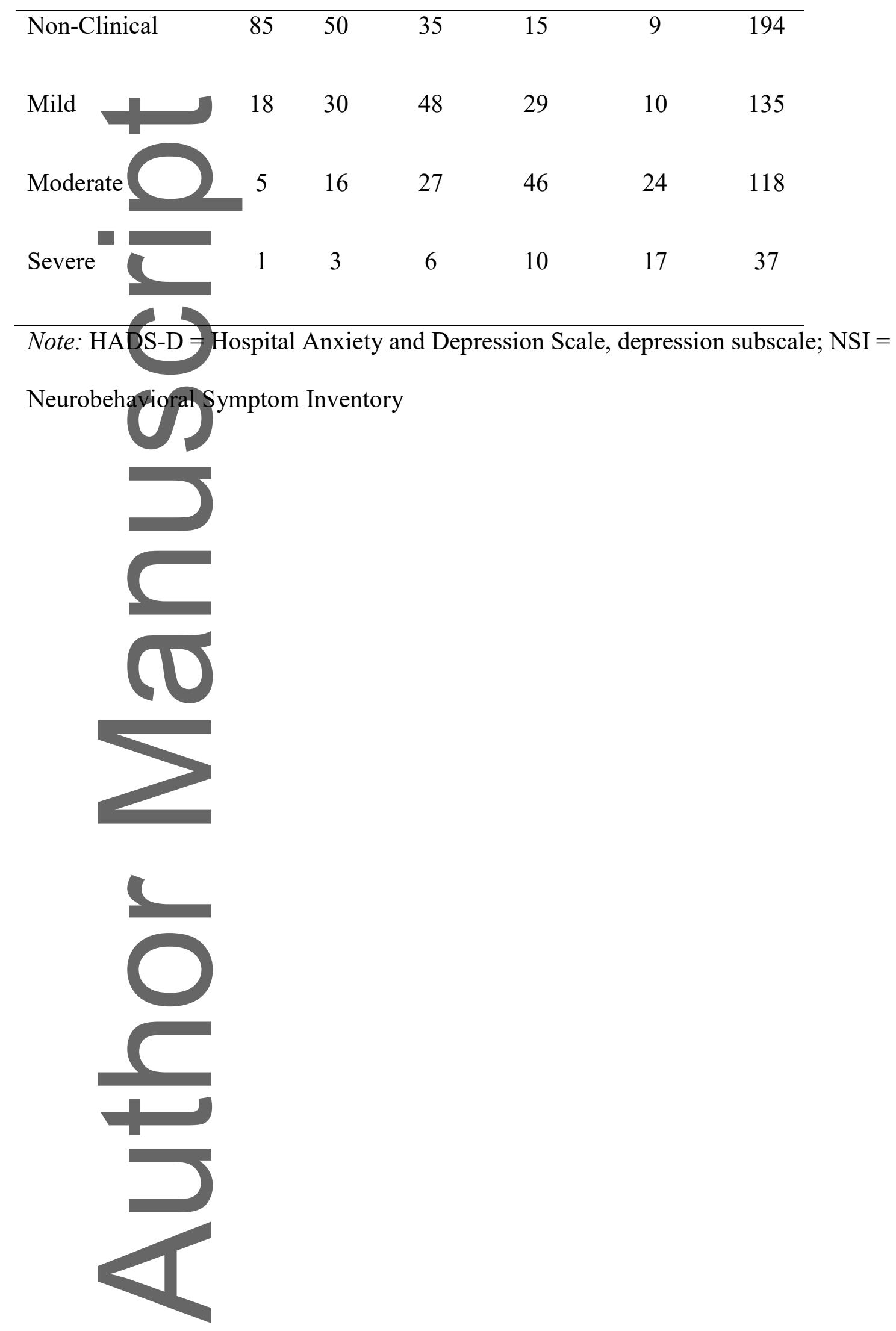

This article is protected by copyright. All rights reserved. 